\title{
The Role of Positron Polarization for the initial 250 GeV stage of the ILC
}

\author{
Jürgen Reuter* \\ on behalf of the Physics Working Group of the LCC Collaboration \\ DESY, Hamburg, Germany \\ E-mail: juergen.reuter@desy.de
}

The International Linear Collider is now proposed with a staged machine design, with the first stage at $\sqrt{s}=250 \mathrm{GeV}$ and an integrated luminosity goal of $2 \mathrm{ab}^{-1}$. One of the questions for the machine design is the importance of positron polarization. In this report, we review the impact of positron polarization on the physics goals of the $250 \mathrm{GeV}$ stage of the ILC and demonstrate that positron polarization has distinct advantages.

39th International Conference on High Energy Physics

4-11 July 2018

Seoul, Korea

\footnotetext{
${ }^{*}$ Speaker.
} 


\section{Introduction}

The International Linear Collider (ILC) is a linear $\mathrm{e}^{+} \mathrm{e}^{-}$collider [1] with a staged operation plan: its initial stage has a center-of-mass energy of $250 \mathrm{GeV}$ (20 km length) [2] and will deliver an integrated luminosity of up to $250 \mathrm{fb}^{-1} /$ year. Later upgrade options include energies of 350 $\mathrm{GeV}, 500 \mathrm{GeV}$ (31 km length) or even $1 \mathrm{TeV}$ (50 km length). The ILC is based on superconducting $\mathrm{RF}$ cavities with $31.5 \mathrm{MV} / \mathrm{m}$ design gradient. In the baseline design, beams with $80 \%$ electron polarization and $30 \%$ positron polarization are foreseen. The project is at the moment under consideration by the Japanese government with a proposed cite in Kitakami in the Iwate prefecture in Northern Japan. The ILC as an electron-positron-collider offers an incredible physics program due to the very clean experimental setup with a well-defined initial state, pure electroweak production and a triggerless operation. This program comprises per-cent and even per-mil-level measurements in the Higgs and top sectors with the possibilities for discoveries of physics beyond the Standard model. The ILC offers the only option to search for light electroweak states that are elusive at hadron colliders. The physics case for the ILC has been summarized by the LCC Physics Working Group in [3, 4] and has been updated for the staged scenario in [5]. A polarized electron beam with up to $80 \%$ polarization is part of the baseline design in every case. In the latest staging report [6], there are two different technological options for the positron source: (1) according to the TDR baseline design, a polarized source using a super-conducting helical undulator of $231 \mathrm{~m}$ length for a $125 \mathrm{GeV}$ electron beam producing a highly collimated polarized photon beam shot on a rotating target, which creates polarized positrons. A spin-rotating solenoid allows any rotation of the polarization vector, in particular both longitudinal and also transversal polarization modes. This option needs the full $125 \mathrm{GeV}$ beam for commissioning. (2) the alternative, more "conventional" design uses a normal-conducting linear accelerator. This option produces unpolarized positrons only. It does not need the full electron beam energy for commissioning, however, leads to a more complicated bunch structure, which possibly needs a second positron damping ring. The technology choice will most likely be made only after an approval of the machine. In this contribution, we discuss why $\mathrm{e}^{+}$polarization is such an important asset of the ILC physics program.

\section{The Surplus of Positron Polarization}

The two main rationales of polarization are higher rates for signal samples, which saves on running time and operation costs, and it enhances signal-to-background ratios by simultaneously suppressing backgrounds. We will first give a general argument for the enhancement of the physics potential with $\mathrm{e}^{+}$polarization, and will then discuss more specific examples.

Beam polarization fractions $\left(\mathscr{P}_{\mathrm{e}^{-}}, \mathscr{P}_{\mathrm{e}^{+}}\right)$are defined as the difference of right and left-handed positron numbers normalized to the sum. First, cross section rates are proportional to the effective luminosity, given in terms of the beam luminosity as $\mathscr{L}_{\text {eff }}:=\frac{1}{2}\left(1-\mathscr{P}_{\mathrm{e}^{-}} \mathscr{P}_{\mathrm{e}^{+}}\right) \mathscr{L}$. For the default ILC setup, $\mathscr{P}_{\mathrm{e}^{-}}=-0.8, \mathscr{P}_{\mathrm{e}^{+}}=+0.3$, this is $0.62 \mathscr{L}$, while it drops to $0.5 \mathscr{L}$ without $\mathrm{e}^{+}$polarization, which amounts to $24 \%$ loss in luminosity. Second, for the most powerful model-independent searches for physics beyond the SM, by parameterizing deviations from the SM in terms of dimension-6 operators in an Effective Field Theory (EFT) expansion, the resolution power for its operator coefficients is greatly enhanced by looking for left-right asymmetries, 
$A_{\mathrm{LR}}=\left(\sigma_{\mathrm{LR}}-\sigma_{\mathrm{RL}}\right) /\left(\sigma_{\mathrm{LR}}+\sigma_{\mathrm{RL}}\right)$. The crucial quantity in these measurements is the effective polarization, $\mathscr{P}_{\text {eff }}=\left(\mathscr{P}_{\mathrm{e}^{-}}-\mathscr{P}_{\mathrm{e}^{+}}\right) /\left(1-\mathscr{P}_{\mathrm{e}^{-}} \mathscr{P}_{\mathrm{e}^{+}}\right)$, whose drop from -0.89 for the standard ILC setup to -0.8 without $\mathrm{e}^{+}$polarization translates to a $10 \%$ loss of analyzing power for the $A_{\mathrm{LR}}$. Besides the increase of $\mathscr{P}_{\text {eff }}$ with $\mathrm{e}^{+}$polarization, the latter also reduces the sensitivity of $\mathscr{P}_{\text {eff }}$ to beam polarization uncertainties. The role of $\mathrm{e}^{+}$polarization in $\mathrm{e}^{-} \mathrm{e}^{+}$collisions is reviewed in [7].

Important physics examples degraded without $\mathrm{e}^{+}$polarization are (1) $s$-channel processes $(\gamma / Z$ exchange) where only $\sigma_{\mathrm{LR}}$ and $\sigma_{\mathrm{RL}} \neq 0$. Hence, using the unpolarized cross section, $\sigma_{0}$, the polarized cross section is $\sigma\left(\mathscr{P}_{\mathrm{e}^{-}}, \mathscr{P}_{\mathrm{e}^{+}}\right)=2 \sigma_{0} \mathscr{L}_{\text {eff }} / \mathscr{L}\left[1-\mathscr{P}_{\text {eff }} A_{\mathrm{LR}}\right]$. The Higgsstrahlung process and hence the whole $250 \mathrm{GeV}$ Higgs program as well as di-fermion processes like $\mathrm{e}^{-} \mathrm{e}^{+} \rightarrow \mathrm{b} \overline{\mathrm{b}}$ are in this category; then, (2) $t$-channel $W / v$ exchange for which only $\sigma_{\mathrm{LR}} \neq 0$, and $\sigma\left(\mathscr{P}_{\mathrm{e}^{-}}, \mathscr{P}_{\mathrm{e}^{+}}\right)=$ $2 \sigma_{0} \mathscr{L}_{\text {eff }} / \mathscr{L}\left[1-\mathscr{P}_{\text {eff }}\right]$. With $\mathrm{e}^{+}$polarization, these processes, $W^{+} W^{-}$production and vector boson fusion into Higgs, have 30\% larger cross section and also 30\% less background. Likewise, a process like single- $W$ production (which allows a very precise determination of the $W$ mass) has only one non-vanishing polarization in either the initial electron or positron (depending on the sign of the $W$ ), and profits similarly from $\mathrm{e}^{+}$polarization. In direct BSM searches, all four polarization combinations can contribute, such that there are more varieties, discussed below.

For a detailed quantization of the surplus of $\mathrm{e}^{+}$polarization, Ref. [5] assumed two scenarios for $2 \mathrm{ab}^{-1}$ at $250 \mathrm{GeV}$, "e $\mathrm{e}^{+}$pol." with $\left|\mathscr{P}_{\mathrm{e}^{-}}\right|=80 \%,\left|\mathscr{P}_{\mathrm{e}^{+}}\right|=30 \%$ and a running scenario with $45 \%$ in the $\left(\mathrm{e}^{-}, \mathrm{e}^{+}\right)$polarization configuration $(+,-), 45 \%$ in $(-,+)$, and $5 \%$ in each of $(+,+)$ and $(-,-)$, respectively. This is compared to the scenario "no e ${ }^{+}$pol." with $\left|\mathscr{P}_{\mathrm{e}^{-}}\right|=80 \%$, $\left|\mathscr{P}_{\mathrm{e}^{+}}\right|=0 \%$ and a running scenario with $50 \%$ each in $(+, \cdot)$ and in $(-, \cdot)$, respectively $(\cdot \equiv$ unpol.). In general, a combination of data samples with different polarizations gives a higher sensitivity than a single sample, it allows to study like-sign polarization sets, and samples with different $\mathrm{e}^{+}$polarization allow for significant reduction of systematic uncertainties by using four different data sets (even nine different sets including transversal polarization).

We now give three examples to show the relevance of $\mathrm{e}^{+}$polarization in SM precision measurements: (1) for a minimization of systematic uncertainties of total cross section and left-right asymmetry measurements, $\mathrm{e}^{+}$polarization is crucial. While electron polarization can always be determined to the sub-permil level, without $\mathrm{e}^{+}$polarization systematic uncertainties in Higgs measurements are up to a factor of five larger without $\mathrm{e}^{+}$polarization [8]. Even when using positron polarimeters, the necessity to experimentally confirm vanishing $\mathrm{e}^{+}$polarization leads to a factor of two to three larger uncertainties for single- $W, W W$ measurements and two-fermion measurements. An independent consistency check on systematics is only possible with $\mathrm{e}^{+}$polarizations. (2) For the search for deviations from the SM in terms of an EFT, in $\mathrm{e}^{-} \mathrm{e}^{+} \rightarrow W^{+} W^{-}$, the most general EFT in that measurement has 14 complex parameters, which can only be extracted using $\mathrm{e}^{+}$polarization. Also, the EFT Wilson coefficient determination of trilinear couplings in $\mathrm{e}^{-} \mathrm{e}^{+} \rightarrow Z \gamma$ needs $\mathrm{e}^{+}$polarization, so measurements of of general SMEFT coefficients are only possible with $\mathrm{e}^{+}$polarization. (3) As a third example, we discuss the importance of $\mathrm{e}^{+}$polarization in the Higgs precision program of the ILC, where it enhances the $Z H$ cross section from 420,000 to 500,000 Higgs bosons with $2 \mathrm{ab}^{-1}$ at $250 \mathrm{GeV}$, which is a reduction in running costs by roughly $19 \%$. For the EFT Higgs coupling fit, having no $\mathrm{e}^{+}$polarization amounts to a degradation of up to $6 \%$, mainly from statistics [9]. For the systematics, the luminosity uncertainty depends much on the $\mathrm{e}^{+}$ 
polarization, again, for the polarization uncertainty, there is a bias from the polarimeter, and different polarization samples allow an in-situ reduction of background uncertainty by a determination of the background from the polarization combinations, which disfavors the signal. In addition, $\mathrm{e}^{+}$ polarization allows new tests of the whole EFT framework by overconstraining the setup.

Finally, we discuss the impact of $\mathrm{e}^{+}$polarization on BSM searches. There is a general paradigm, that a polarized positron source could be built after a possible new physics discovery for discrimination, however, such a separation is not necessarily possible. Most likely, a large data set is needed to establish a $3 \sigma$ evidence or $5 \sigma$ discovery, and $\mathrm{e}^{+}$polarization would very early on provide a handle on the significance. Discoveries could even be missed without $\mathrm{e}^{+}$polarization: examples are contact interactions $\left(Z^{\prime}\right)$, where $\mathrm{e}^{+}$polarization enhances the reach for new physics scales by $30 \%$ by using four different polarization samples, the search for heavy leptons in $\mathrm{e}^{-} \mathrm{e}^{+} \rightarrow W^{+} W^{-}$needs double polarization asymmetries, which is only possible with $\mathrm{e}^{+}$polarization, and the search for light pseudo-Nambu-Goldstone bosons in $\mathrm{e}^{-} \mathrm{e}^{+} \rightarrow \mathrm{b} \bar{b} \phi$ needs $\mathrm{e}^{+}$polarization for a determination of the quantum numbers [10], and in the search for invisible particles the possibility of using LR/RL versus LL/RR samples allows to separate signal from backgrounds, and in EFT dark matter searches $\mathrm{e}^{+}$polarization saves up to 2.5 years of run time.

\section{Conclusions}

The ILC in its $250 \mathrm{GeV}$ stage offers an indispensable physics program with its high-precision Higgs program, model-independent searches of electroweak states and direct dark matter searches. Positron polarization crucially enhances the physics potential by an improvement of signal-tobackground ratios, a safeguard against systematic uncertainties and by allowing much higher sensitivity through a combination of different polarization data samples. Several of these points lead to savings on running costs of up to $20 \%$. These issues should be carefully considered when the technology choice for the positron source is made.

\section{References}

[1] T. Behnke et al., arXiv:1306.6327 [physics.acc-ph]; H. Baer et al., arXiv:1306.6352 [hep-ph]; C. Adolphsen et al., arXiv:1306.6353 [physics.acc-ph]; C. Adolphsen et al., arXiv:1306.6328 [physics.acc-ph]; T. Behnke et al., arXiv:1306.6329 [physics.ins-det].

[2] T. Barklow, J. Brau, K. Fujii, J. Gao, J. List, N. Walker and K. Yokoya, arXiv:1506.07830 [hep-ex].

[3] K. Fujii et al., arXiv:1506.05992 [hep-ex].

[4] K. Fujii et al., arXiv:1702.05333 [hep-ph].

[5] K. Fujii et al., arXiv:1710.07621 [hep-ex].

[6] L. Evans et al. [Linear Collider Collaboration], arXiv:1711.00568 [physics.acc-ph].

[7] G. Moortgat-Pick et al., Phys. Rept. 460, 131 (2008) [hep-ph/0507011].

[8] R. Karl. 2018, PhD thesis in preparation, University of Hamburg.

[9] T. Barklow et al., Phys. Rev. D 97, no. 5, 053003 (2018) [arXiv:1708.08912 [hep-ph]].

[10] W. Kilian, D. Rainwater and J. Reuter, Phys. Rev. D 71, 015008 (2005) [hep-ph/0411213]; Phys. Rev. D 74, 095003 (2006) Erratum: [Phys. Rev. D 74, 099905 (2006)] [hep-ph/0609119]. 